$-120-$

\section{FACTORS THAT EXACERBATE ANGINA PECTORIS AFTER ABRUPT WITHDRAWAL OF ANTIANGINAL DRUGS}

Miwa Atsuko, Kawakubo Kiyoshi, Toda Iku, Murakawa Yūji, Nakajima Toshiaki, Nozaki Akira, Kurachi Yoshihisa, Ooshiro Masaya, Kawahara Takashi, Iizuka Masahiko, Mashima Saburo, Sugimoto Tsuneaki.

Second Department of Internal Medicine, Faculty of Medicine, University of Tokyo. Factors contributing to the worsening of angina after abrupt withdrawal of antianginal drugs were studied. Method: Seventy eight patients with angina pectoris, median age 56 years have experienced withdrawal of antianginal drugs. In these patients the review of medication and efficacy of treatment were evaluated. Rebound was defined as the return of anginal symptoms to levels exceeding those at pretreatment base line state. Results: Fourteen(18 $\%$ ) patients exhibited rebound of the symptoms, in 4 of whom myocardial infarction developed after drug withdrawal. The rebound was observed in 7(50 $\%)$ patients after withdrawal of $\mathrm{Ca}$ antagonists and in $12(86 \%)$ after withdrawal of drugs including $\mathrm{Ca}$ antagonists. The rebound after $\beta$-blocker withdrawal was noted in only 1 patient. The rebound group consisted of more patients having angina at both rest and effort, variant angina, and one vessel disease than the without rebound group $(\mathrm{p}<0.01,0.0$ $1,0.05)$. Seven( $32 \%)$. of 22 patients with Ca antagonist withdrawal showed rebound, and 6 of 7 had variant angina. Ten (71\%) of 14 patients showed rebound in whom the drugs were withdrawn within one year after the onset of angina. The rebound occurred more frequently in the patients who were asymptomatic for less than one month before drug withdrawal $(\mathrm{p}<0.01)$. Al1 4 patients who experienced acute myocardial infarction had variant angina and rebound occurred after $\mathrm{Ca}$ antagonist withdrawal. Conclusions: Rebound of anginal symptoms should be considered after the withdrawal of antianginal drugs, particularly $\mathrm{Ca}$ antagonists, in those with angina of vasospastic nature and of recent onset.

\section{$-121-$ \\ ACUTE HEMODYNAMIC EFFECTS OF INTRA- VENOUS DILTIAZEM AND NITROGLYCERIN ALONE AND IN COMBINATION IN PATIENTS WITH CORONARY ARTERY DISEASE}

Hiroshi AMEMIYA, Hideomi FUJIWARA, Nobuhiro SHIROYAMA, Shigeo UMEZAWA, Yoshito IESAKA and Koichi TANIGUCHI *

Division of Cardiology,

Tsuchiura Kyodo General Hospital.

The Second Department of Internal Medicine, Tokyo Medical and Dental University*.

Acute coronary and left ventricular hemodynamic effects of intravenous diltiazem and nitroglycerin in combination were evaluated in 35 patients with coronary artery disease. These results were compared with those in 15 patients given diltiazem initially and 15 patients nitroglycerin initially. The diltiazem-nitroglycerin combination

significantly reduced heart rate $(H R, p<0.05)$, mean aortic pressure(MAP, $p<0.001)$, left ventricular end-diastolic pressure (LVEDP, $p<0.001$ ), cardiac index $(C I, p<0.05)$, stroke work index(SWI, $p<0.001)$, pressure-rate product (PRP, $p<0.001)$, systemic vascular resistance(SVR, $\mathrm{p}<0.001$ ) and coronary vascular resistance (CVR, $p<0.01)$. Coronary sinus flow(CSF) and stroke index(SI) were both maintained.

To compare with nitroglycerin alone, these combination suppressed the increase of $H R$, the decrease of $\mathrm{CI}$ and $\mathrm{SI}$ sustaining the decrease of LVEDP, and induced the decrease of MAP, SVR, CVR and PRP.

Then comparing with diltiazem alone, these combination caused the significant decrease of IVEDP and SWI, maintaining the decrease of HR, PRP and SVR.

Then for the combination group, MAP and PRP rather decreased in comparison with each drug alone, both the decrease of CSF and the increase of CVR were not obserbed, so these findings suggest the possibility of improving the balance of myocardial oxygen supply and demand.

In conclusion, the results of this study suggest that the favorable propaties of each drug are comparatively retained and the unwanted effects suppressed in diltiazem-nitroglycerin combination.

\section{$-122-$}

EFFECTS OF NITROGLYCERIN ON SYMPATHETIC NERVE ACTIVITY IN ANESTHETIZED RABBITS

Kenichi Kujime, Yumi Namikata, Hisayuki

Sawatari, Kazumi Maeda, Yasuyuki Ueba

School of Allied Medical Sciences, Kobe University

Nitroglycerin(GTN) is a potent hypotensive drug which has a direct vasodilating action on blood vessels. Although sympathetic nerve activity (SNA) has protective effects on hypotension, it is still unclear how GTN exerts its effects on baroreflex or SNA.

In anesthetized rabbits, renal sympathetic nerve activity (RNA) and arterial pressure (AP) were recorded simultaneously. GTN was injected intravenously at a rate of $12,30,60 \mathrm{ug} / \mathrm{kg} / \mathrm{min}$ for 6 to $10 \mathrm{~min}$. GTN decreased AP by $16,25,39 \%$ while RNA dropped by $3,18,27 \%$ in dose of 12 $30,60 \mathrm{ug}$, respectively. The correlation between $A P$ and RNA was measured during artificial changes of AP induced by inflation of a balloon in the descending aorta. This showed that GTN made baroreflex less sensitive dose-dependently $(y=-x+1$ in control, $y=-x+0.83$ in 30 ug. $y=-0.85 x+0.68$ in $60 \mathrm{ug})$. In order to clarify the mechanism, bilateral vagus nerve and/or carotid sinus nerve(CSN) were severed. GTN dropped RNA by $6,9,12 \%$ after the single denervation of CSNs, and by $1,4,8 \%$ after vagotomy alone, in dose of 12, 30, $60 \mathrm{ug}$, respectively. But, after total denevation of the two sets of the nerves, GTN did not induce a fall in RNA any longer.

These results suggest that GTN has an influence on SNA by desensitization on baroreflex through both vagus nerve and CSN. Thus, GTN has the effects on AP by indirect action on blood vessels as well as direct action.

\section{$-123-$}

ALTERNATION IN LEFT VENTRICULAR FUNCTION AND REFLEX SYMPATHETIC RESPONSE WITH DRIP INFUSION OF NITROGLYCERINE

Akira Miyamoto, Kyoichi Mizuno, Bonpei Takase, Ko Arakawa, Toshio Shibuya, Kazushige Isojima, Toshiharu Maruyama, Kenji Horiuchi, Akira Kurita,

Haruo Nakamura. 